Proceedings

\title{
Twisted Fibers Can Have an Adjustable Thermal Expansion ${ }^{\dagger}$
}

\author{
Donghua Yue * and Liming Wei \\ Institute of Systems Engineering, China Academy of Engineering Physics, Mianyang 621999, China; \\ 401weilm@caep.cn \\ * Correspondence: 1xyuedh@caep.cn; Tel.: +86-816-2494-848 \\ + Presented at the 18th International Conference on Experimental Mechanics (ICEM18), Brussels, Belgium, \\ 1-5 July 2018.
}

Published: 13 June 2018

\begin{abstract}
In this paper, a device with high accuracy capacitive sensor (with the error of 0.1 micrometer) is constructed to measure the axial thermal expansion coefficent of the twisted carbon fibers and yarns of Kevlar. A theoretical model based on the thermal elasticity and the geometrical features of the twisted structure is also presented to predict the axial expansion coefficient. It is found that the twist angle, diameter and pitch have remarkable influences on the axial thermal expansion coefficients of the twisted carbon fibers and Kevlar strands, and the calculated results are in good agreement with experimental data. We found that, with the increase of the twist angle, the absolute value of the axial thermal expansion coefficient increases. For the Kevlar samples, the expansion coefficient will grow by about $46 \%$ when the twist angle increases from 0 to 25 degrees, while the carbon fiber samples will grow by about $72 \%$ when the twist angle increases from 0 to 35 degrees. The experimental measurements and the model calculations reveal important properties of the thermal expansion in the twisted structures. Most notably, the expansion of the strand during heating or cooling can be zero when the twist angle is around $\beta=\arcsin (\alpha \mathrm{L} / \alpha \mathrm{T})^{\wedge} 1 / 2$, where $\beta$ denotes twist angle of the strand and $\alpha \mathrm{L}, \alpha \mathrm{T}$ are the longitute and the transverse thermal expansion coefficient of the strand, respectively. According to the present experiments and analyses, a method to control the axial thermal expansion coefficient of this new kind of twisted structure is proposed. Moreover, the mechanism of this tunable thermal expansion is discussed. Based on the model, a method that can be used to rectify the thermal expansion properties of the twist structures is established. This may be a new way of fabricating zero expansion composite materials in the future.
\end{abstract}

Keywords: twisted structure; carbon fibre; tunable thermal expansion coefficient

\section{Introduction}

The coefficient of thermal expansion (CTE) is one of the most important parameter of thermal physical properties. Materials and structures with adjustable thermal expansion are urgently needed in engineering applications, especially in precision instruments, aerospace, and civil engineering, where the control of thermal expansion and stress due to a wide range of temperatures is very important [1]. Therefore, the development of materials or structures with tunable thermal expansion has important scientific significance and engineering applications. The new concept of zero thermal expansion rope twisted by polymers and carbon fibers has been investigated hereinaccording to its distinguishing advantages e.g., high energy density, large-stroke, non-hysteresis, and inexpansiveness [2,3]. The structure of twisted wire is widely used in engineering of electric cables, bridges, drilling platforms and elsewhere. That is because of their ability to resist axial load and flexible in bending [4]. It is important to test and calculate the thermal expansion exactly. There are 
several key factors that have a strong impact on the thermal expansion of twisted wire. The pitch of the strand and contact force mean that even the friction can change the thermal expansion characteristics of the twisted-wire. The fabrication process of this kind of artificial muscle is very simple and convenient, the deformation principle of which is in response to the thermal expansion.

\section{Experiment}

In this paper, a device with high accuracy capacitive sensor (with the error of 0.1 micrometer) is constructed to measure the axial thermal expansion coefficent of the twisted carbon fibers and yarns of Kevlar. The twisted-pair strand was formed by two bundles of fibers, with the angle of $\alpha=12^{\circ} / 13^{\circ} / 17^{\circ} / 25^{\circ}$ for the Kevlar strand and $\alpha=10^{\circ} / 18^{\circ} / 31^{\circ} / 35^{\circ}$ for the carbon strand. The twisted angle of the strand was obtained by Equation (1):

$$
\alpha=\arctan \left(\frac{\pi \mathrm{D}}{h}\right)
$$

where $\alpha$ denotes twist angle of the strand, $\mathrm{h}$ is the pitch of the strand, and $\mathrm{D}$ is the Diameter of the bundles. The diameter of the carbon fiber bundles and strands were $1 \mathrm{~mm}$ and $2 \mathrm{~mm}$, respectively. The diameter of the Kevlar fiber bundles and strands were $0.45 \mathrm{~mm}$ and $0.9 \mathrm{~mm}$, and the length of the samples was about $250 \mathrm{~mm}$. The experiments were performed at room temperature $20^{\circ} \mathrm{C} ; 25 \%$ of the elastic limit was chosen as the preload. The slope of the displacement and temperature curve was used to calculate the thermal expansion coefficient. A self-made device for measuring the axial thermal expansion coefficient of parallel fiber and a two-ply strand was constructed by using the capacitive displacement sensor inside a quartz tube wrapped in heating wire, as shown in Figure 1.

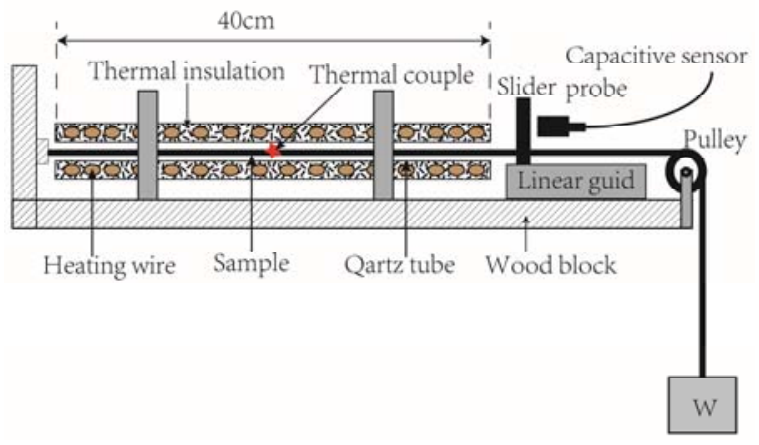

Figure 1. Schematic of the apparatus used to measure the axial thermal expansion coefficient.

The test system was fixed on the damping platform. The effect of the friction in the guide rail and the pulley can be neglected as the force is so small compared to the hanging weight. A thermometer has been slipped along every centimeter to measure the temperature along the tube. The temperature change profile is shown in Figure 2.

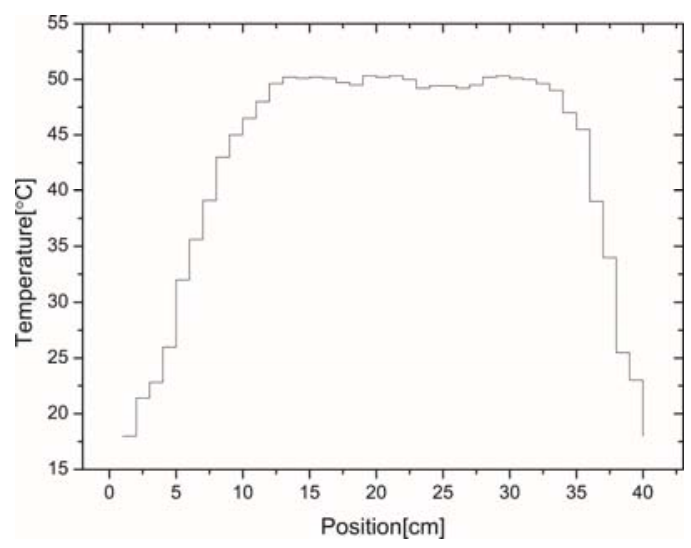

Figure 2. Temperature distribution in the heating tube. 
Temperature fluctuation in the tube are the main sources of error in the experiment at the level of roughly $\pm 0.6^{\circ} \mathrm{C}$. The temperature change profile was then used to calculate the length of the effective homogeneous area. The effective homogeneous length was $32 \mathrm{~cm}$ with the equivalent elongation of integral divided into $1 \mathrm{~cm}$ sections. The thermal expansion coefficient of sample is defined as:

$$
\alpha_{\text {eff }}=\frac{\Delta L}{L_{\text {eff }}\left(T_{\text {High }}-T_{\text {Low }}\right)}
$$

where $\Delta L$ is the contraction of the samples when the temperature rises, and $T_{\text {High }}$ and $T_{\text {Low }}$ are the heating temperature and initial temperatures, respectively. The effective length of the homogeneous area can be expressed by:

$$
L_{\text {eff }}=\int_{0}^{L}\left(T-T_{\text {Low }}\right) d L /\left(T_{\text {High }}-T_{\text {Low }}\right)
$$

This assumption will not affect the integrity of the results calculated in this paper. The previous results show that when the thermal expansion of carbon fiber was measured at around $25^{\circ} \mathrm{C}$, the thermal expansion is $-1.17 \times 10^{-6} \mathrm{~K}^{-1}$. The calculated thermal expansion is $-1.2 \times 10^{-6} \mathrm{~K}^{-1}$ with the effective length of our test results.

\section{Results and Discussion}

The results of the carbon sample are shown in Figure 3a,b, where the twisted angle is $\alpha=31^{\circ}$. The contractions of the Kevlar parallel fibers and strand with the twist angle $\alpha=25^{\circ}$ are shown in Figure $4 a, b$.

The thermomechanical mechanism is considered to have qualified the thermal stress in the strand when being heated. A theoretical model was obtained to quantify the axial expansibility of the strand twisted by anisotropic fibers. The coefficient of thermal expansion of the strand can be defined as:

$$
\alpha_{e f f}=\frac{\alpha_{L}-\alpha_{\mathrm{T}} \sin ^{2} \alpha}{\cos ^{2} \alpha}
$$

A comparison of theoretical and experimental effective thermal expansion coefficient against the twist angle is shown in Figure 5. The dot symbols denote the thermal expansion coefficients of the test results. The dot-lines display the calculated results according to the above module. From this equation we can predict the giant contraction in strand resulting from the anisotropic thermal expansion coefficients. One can see that the theoretical results are in good agreement with the experimental results.
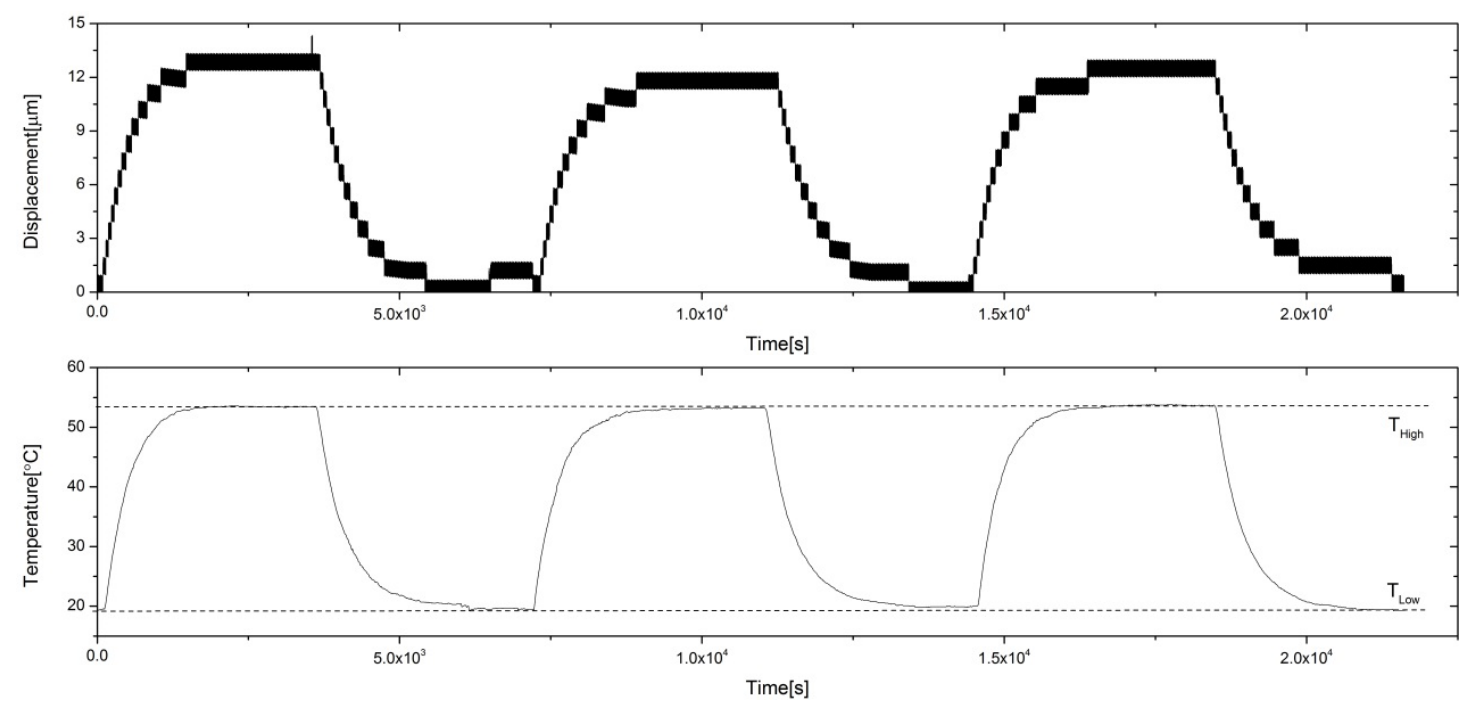

(a) 

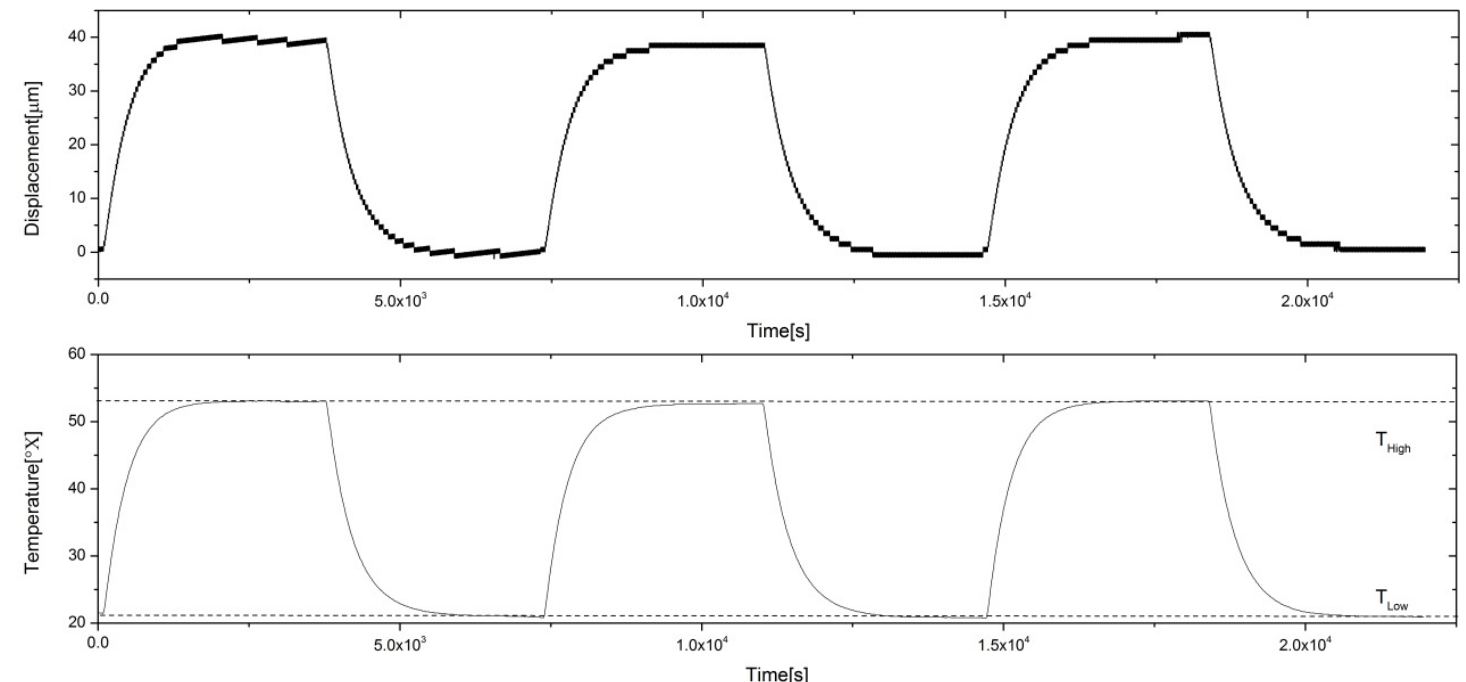

(b)

Figure 3. (a) Contraction of the carbon fiber bundle with the twist angle $\alpha=0$; (b) contraction of the carbon fiber strand with the twist angle $\alpha=31^{\circ}$.
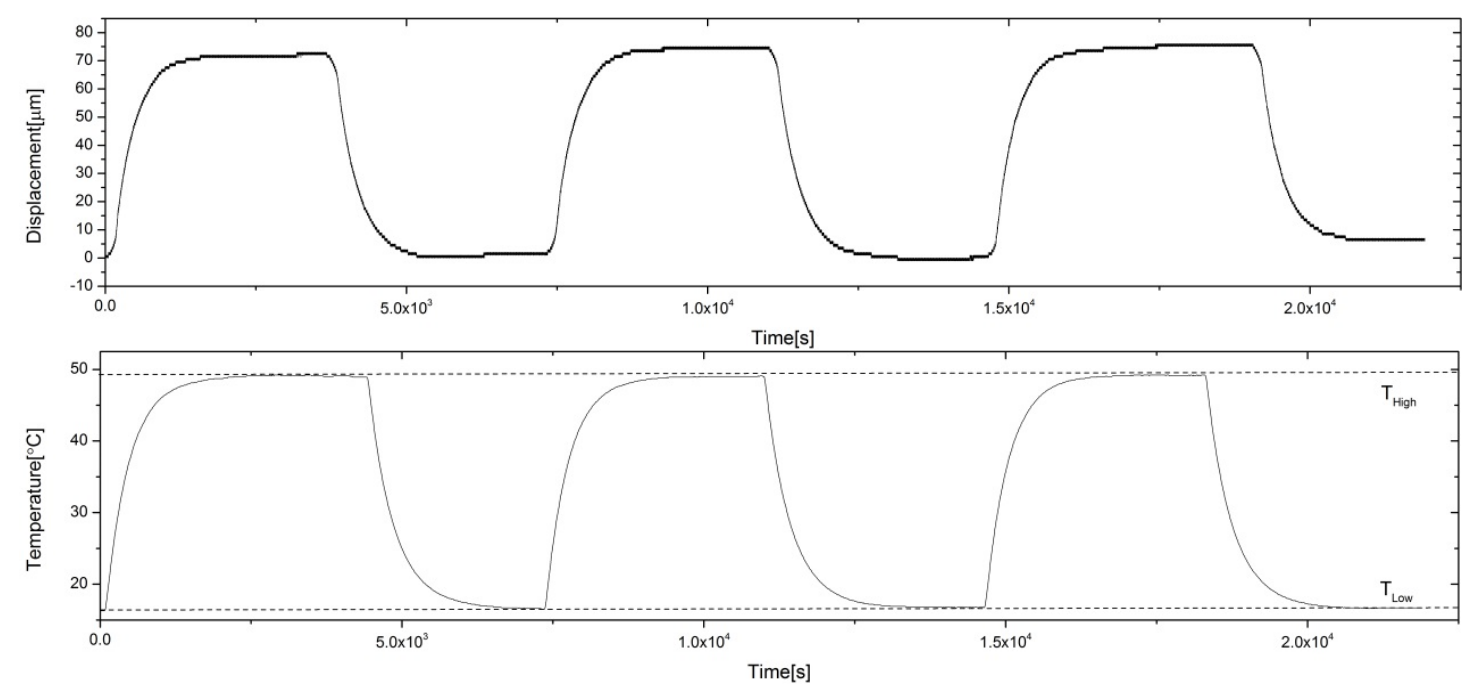

(a)
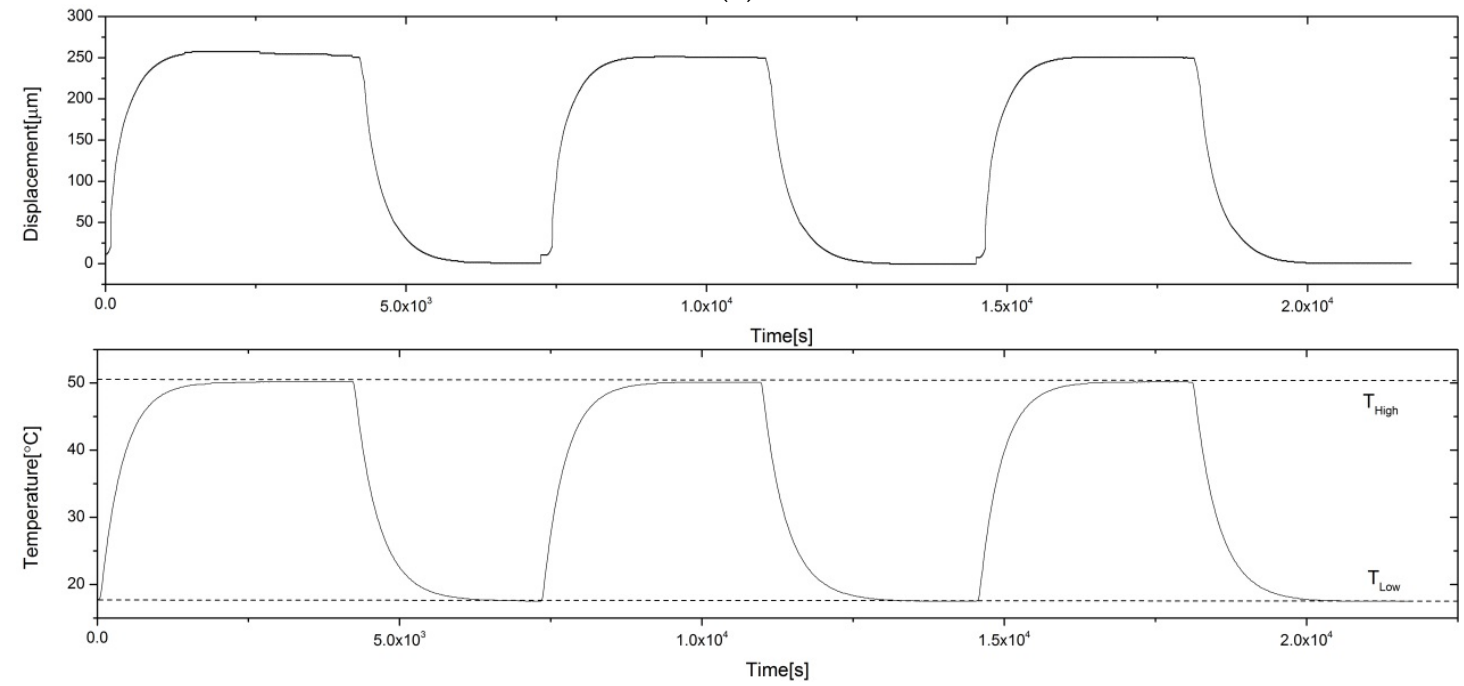

(b)

Figure 4. (a) Contraction of the Kevlar-29 fiber bundle with the twist angle $\alpha=0^{\circ}$; (b) contraction of the carbon fiber strand with the twist angle $\alpha=25^{\circ}$. 


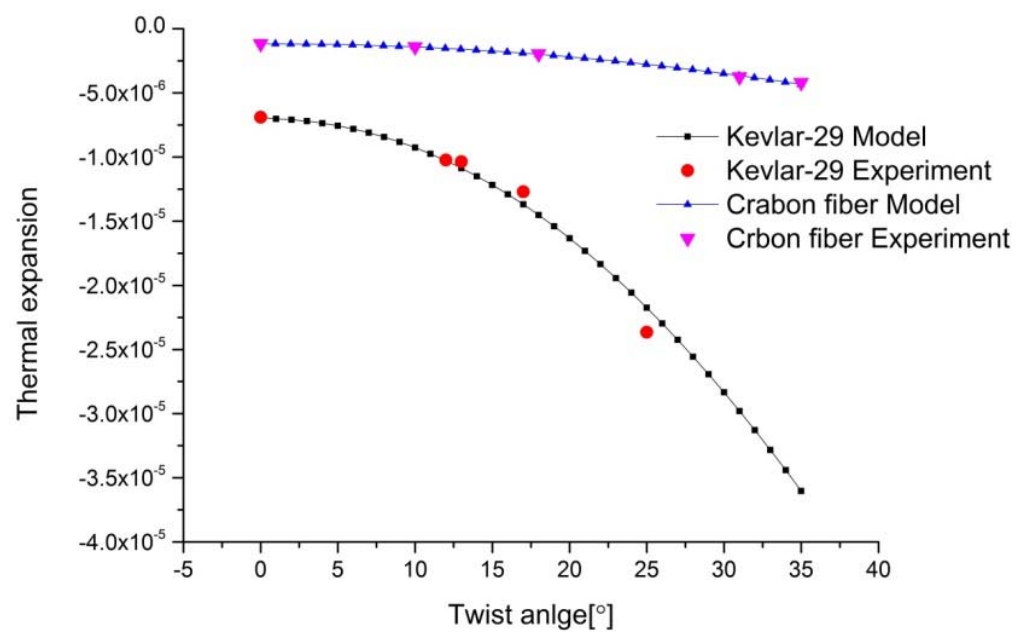

Figure 5. The adjustable axial thermal expansion coefficient of the strand varies with the twist angle.

\section{Conclusion}

In summary, the axial thermal expansion coefficient of the twisted-pair strand during heating and cooling were measured. It was found that the coefficient of the amplification due to twisting was associated with two factors: the degree of twisting and the degree of anisotropy of the thermal expansion coefficient. The relationships between degree of twisting and axial thermal expansion coefficient have been obtained and verified by an experiment with a Kevlar and carbon fiber strand. According to the theoretical analysis, we can obtain a more contractive rope by twisting the strands together. Therefore, the expansion of the strand during heating or cooling can be zero when the twist angle is around $\beta=\arcsin \sqrt{\alpha_{L} / \alpha_{T}}$. This is a new way of fabricating zero-expansion composite materials in the future.

Acknowledgments: This work is supported by the Innovation and Development Fund of the Institute of Systems Engineering (No. 2017 cxj10).

\section{References}

1. Bell, C.J.; Reid, S.; Faller, J.; Hammond, G.D.; Hough, J.; Martin, I.W.; Rowan, S.; Tokmakov, K.V. Experimental results for nulling the effective thermal expansion coefficient of fused silica fibres under a static stress. Class. Quantum Grav. 2014, 31, 065010.

2. Lima, M.D.; Li, N.; De Andrade, M.J.; Fang, S.; Oh, J.; Spinks, G.M.; Kozlov, M.E.; Haines, C.S.; Suh, D.; Foroughi, J.; et al. Electrically, chemically, and photonically powered torsional and tensile actuation of hybrid carbon nanotube yarn muscles. Science 2012, 338, 928-932.

3. Bohr, J.O.K. The ancient art of laying rope. EPL Europhys. Lett. 2011, 93, 60004.

4. Costello, G.A. Theory of Wire Rope; Springer Science \& Business Media: Berlin/Heidelberg, Germany, 1997.

C 2018 by the authors. Licensee MDPI, Basel, Switzerland. This article is an open access article distributed under the terms and conditions of the Creative Commons Attribution (CC BY) license (http://creativecommons.org/licenses/by/4.0/). 